\title{
Parallel circuits for refractory hypoxemia on venovenous extracorporeal membrane oxygenation
}

\author{
Adnan Malik, MD, ${ }^{a}$ Larry L. Shears, MD, ${ }^{b}$ Dmitriy Zubkus, MD, ${ }^{c}$ and David J. Kaczorowski, MD, ${ }^{b}$ \\ York, $\mathrm{Pa}$
}

\author{
From the Departments of ${ }^{\mathrm{a} C}$ Cardiology, ${ }^{\mathrm{b}}$ Cardiothoracic Surgery, and ${ }^{\mathrm{c}}$ Pulmonary and Critical Care Medicine, \\ WellSpan Health, York, Pa. \\ Disclosures: Authors have nothing to disclose with regard to commercial support. \\ Received for publication July 28, 2016; revisions received Sept 28, 2016; accepted for publication Oct 16, 2016; \\ available ahead of print Dec 4, 2016. \\ Address for reprints: David J. Kaczorowski, MD, Cardiothoracic Surgery, WellSpan Health, 25 Monument Rd, \\ Ste 190, York, PA 17403 (E-mail: dkaczorowski@wellspan.org). \\ J Thorac Cardiovasc Surg 2017;153:e49-51 \\ $0022-5223 / \$ 36.00$ \\ Copyright (C) 2016 by The American Association for Thoracic Surgery \\ http://dx.doi.org/10.1016/j.jtcvs.2016.10.067
}

Video clip is available online.

Refractory hypoxemia during support on venovenous (VV) extracorporeal membrane oxygenation (ECMO) is a challenging clinical problem. Manipulating ventilator settings, prone positioning, optimizing cannula position, and ECMO flows, and the use of beta blockers have been advocated as methods to improve oxygenation during $\mathrm{VV}$ ECMO. ${ }^{1,2}$ Cannulation strategies designed to prevent mixing have also been reported. ${ }^{3}$ In patients with extremely high cardiac output, including those with trauma, sepsis, or other systemic inflammatory responses, these strategies may be inadequate or ineffective because the patient's cardiac output may be substantially greater than the maximum flow achievable with an ECMO circuit. Here we report a novel strategy that was used to overcome refractory hypoxemia in a patient with high cardiac output. Specifically, a second ECMO circuit was used in parallel with the first to achieve adequate oxygenation.

\section{CLINICAL SUMMARY}

A 39-year-old woman developed severe hypoxemia due to acute respiratory distress syndrome as a complication of intra-abdominal sepsis. She was placed on VV ECMO using standard techniques (Figure 1, A). Venous drainage was accomplished using a 25-French long, multiholed cannula in the right common femoral vein with its tip at the junction of the inferior vena cava (IVC) and right atrium. An 18-French cannula was placed via the right internal jugular vein with its tip located in the right atrium. ECMO flows of approximately $5.5 \mathrm{~L} / \mathrm{min}$ were achieved and were considered adequate because the patient's weight was

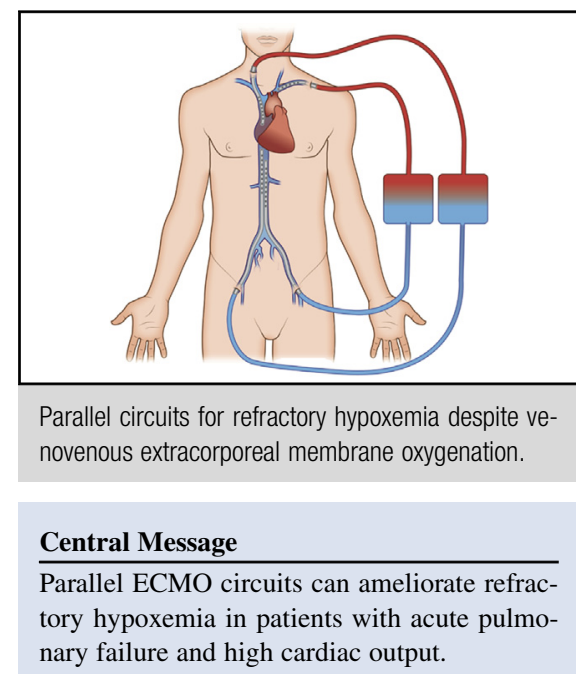

See Editorial Commentary page e53.

$55 \mathrm{~kg}$ and her body surface area was $1.6 \mathrm{~m}^{2}$. The patient improved and adequate oxygenation was achieved.

The patient's condition deteriorated over the next several days. She was tachycardic and had a cardiac output ranging between 8 and $10 \mathrm{~L} / \mathrm{min}$. Despite optimizing ventilator settings and maximizing ECMO flows, the patient's hypoxemia worsened with an arterial partial pressure of oxygen as low as $40 \mathrm{~mm} \mathrm{Hg}$. The position of the cannulas was confirmed with both chest radiograph and echocardiography. Echocardiography showed no evidence of intracardiac shunt. Visual inspection of the arterial and venous lines suggested minimal recirculation and this was supported by blood gas data. Further, the venous drainage cannula was manipulated in an attempt to reduce any potential recirculation without any significant change in oxygenation or circuit flows. Oxygenator problems were also excluded. Visual inspection revealed no evidence of thrombus in the oxygenator. An oxygenator gas test revealed an arterial partial pressure of oxygen of $464 \mathrm{~mm} \mathrm{Hg}$. Beta blockade was not possible due to hypotension. The ability to diurese the patient was limited by the patient's septic state. To better match the patient's high cardiac output, we sought to provide considerably more ECMO flow, beyond what might be achieved by insertion of an additional venous drainage cannula.

A second ECMO circuit was placed in parallel with the first circuit (Figure 1,B). A 21-French long, multiholed 

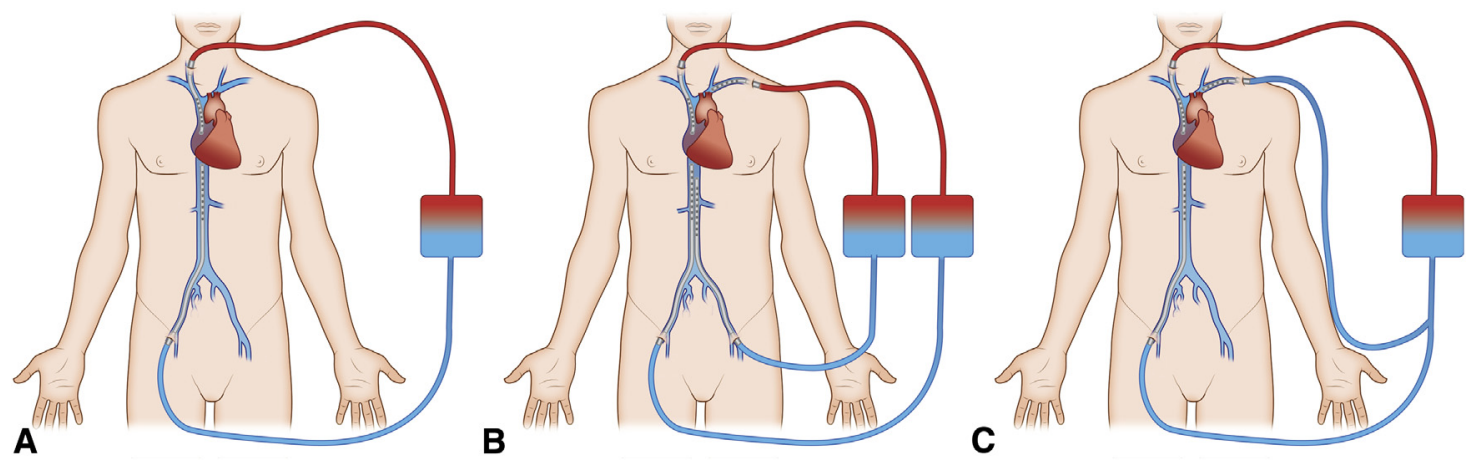

FIGURE 1. Parallel circuits for refractory hypoxemia despite venovenous extracorporeal membrane oxygenation (VV ECMO). A, Standard VV ECMO with cannulation of the right common femoral vein and the right internal jugular vein. B, Parallel VV ECMO circuits with a second circuit involving cannulation of the left common femoral vein and left subclavian vein. C, Conversion back to a single circuit with venous drainage from the both the left subclavian vein and right common femoral vein and return via cannulation of the right internal jugular vein.

venous cannula was placed via the left femoral vein with its tip located more peripherally in the IVC. Another 18-French cannula was placed in the left subclavian vein with its tip near the superior vena cava. ECMO was initiated and the patient's oxygenation improved. After adding the second circuit, flows of $4.75 \mathrm{~L} / \mathrm{min}$ and $3.5 \mathrm{~L} / \mathrm{min}$ were achieved with the first and second circuits, respectively. Approximately 8 to $9 \mathrm{~L} / \mathrm{min}$ combined flow could be delivered at a given moment in this patient. The first arterial blood gas after insertion of the second circuit revealed an arterial partial pressure of oxygen of $92 \mathrm{~mm} \mathrm{Hg}$. Within 12 hours, the arterial partial pressure of oxygen reached $187 \mathrm{~mm} \mathrm{Hg}$. This increase in oxygenation allowed the fraction of inspired oxygen on the ventilator to be weaned from $100 \%$ to $30 \%$ within 24 hours. No other interventions were performed during that period of time that could account for the observed improvement in oxygenation.

The patient was maintained on dual circuits for 14 days. At that time, the left femoral venous cannula was removed. The left subclavian cannula was then connected to the venous limb of the first circuit and used as an additional site of venous drainage (Figure 1,C). Later, a single-site cannulation strategy using a dual-lumen bicaval cannula was used to allow mobility as she improved. The patient was weaned from ECMO after 60 days of support. She had a prolonged intensive care unit stay notable for acute renal failure requiring temporary hemodialysis. She was later discharged from the hospital decannulated of her tracheostomy and with normal renal function.

\section{DISCUSSION}

The use of ECMO for the management of patients with acute cardiac or pulmonary failure has grown substantially in recent years. ${ }^{4}$ Although ECMO can be life saving for appropriately selected patients, those with high cardiac output can be challenging to manage with ECMO and outcomes may be suboptimal, particularly in the setting of sepsis. ${ }^{5}$ This report demonstrates that parallel VV ECMO circuits can be successfully used to treat refractory hypoxemia in the setting of elevated cardiac output in a patient with acute pulmonary failure (Video 1).

One alternative to the approach used here is conversion to venoarterial (VA) ECMO. VA ECMO can be useful to ameliorate both hypotension and ongoing hypoxemia in many cases. This is particularly true if myocardial function is impaired. However, in the setting of extremely high cardiac output and vasodilatation, VA ECMO may be ineffective and potentially deleterious. Because the cardiac output is already high, VA ECMO does not address the underlying pathophysiology and blood pressure may remain low under these circumstances due to lack of vascular tone. Further, when native cardiac output is very high and femoral cannulation is employed, mixing may occur very distally in the aorta, resulting in delivery of deoxygenated blood to the upper body. These may be contributing factors to the poor outcomes observed with the use of VA ECMO in the management of septic shock.

Whereas VA ECMO may be ineffective in patients with hypoxemia and high cardiac output, several other maneuvers should be considered to remedy the situation. Cannula position should be optimized. The venous cannula was pulled back in this case; however, we did not

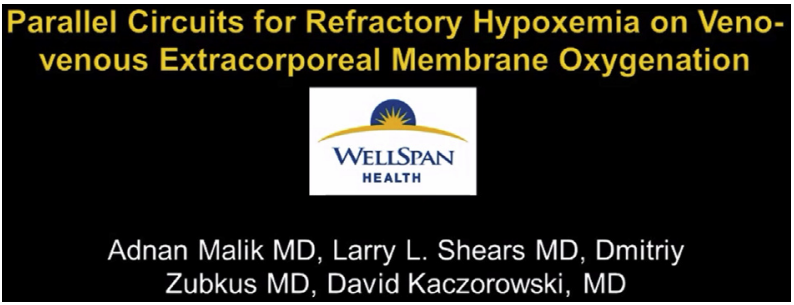

VIDEO 1. Refractory hypoxemia during venovenous extracorporeal membrane oxygenation and the case presented here are discussed. Video available at: http://www.jtcvsonline.org/article/S0022-5223(16)31467-2/ addons. 
manipulate the infusion cannula. It is possible that doing so would have reduced potential recirculation and improved oxygenation. Insertion of a drainage cannula in the superior vena cava is also a viable alternative to consider before using parallel circuits. Because insertion of a superior vena cava cannula may allow additional ECMO flow and reduce mixing, this intervention should be considered before inserting a second circuit. In this case, we sought to provide more flow than what might be expected from the insertion of an additional cannula. Furthermore, the Extracorporeal Life Support Organization provides guidelines on the management of patients receiving ECMO. ${ }^{6}$ Given the important role of hemoglobin in oxygen delivery, these guidelines state that hematocrit level should be targeted as high as $>40 \%{ }^{6}$ Using a higher goal hematocrit level before inserting the second circuit may have allowed for improved oxygen delivery in this case. Another potential alternative to placing a second circuit is prone positioning. Although this is certainly a possibility, we do not routinely place patients on ECMO in the prone position due to concern for potential complications, including cannula malposition and cannula dislodgement.

The use of femoral cannulation in this strategy precludes early mobilization and ambulation. However, it is unlikely that patients requiring parallel circuits would be suitable candidates for extubation or ambulation at that particular stage in their disease. A strategy involving parallel circuits does not preclude later conversion to a single-site, bicaval strategy, as was performed in this case. We did not find that the addition of a second circuit was overly cumbersome for the nursing staff and it did not impair daily nursing care.

The cannulation strategy used in this case required positioning of 2 venous cannulas in the IVC, leading to concern over potential thrombotic complications involving the IVC. Adequate anticoagulation is likely an important factor to prevent these complications. In this case, the patient was systemically anticoagulated with a goal partial thromboplastin time of 60 to 80 seconds, but the ideal degree of anticoagulation is unknown. Greater experience with this technique will be required to evaluate the risk of this and other potential complications.

Another potential concern is the cost of an additional ECMO circuit. In this case, the costs of the disposables used to establish the second circuit were minor. The majority of the cost of care provided stemmed from the patient's prolonged intensive care stay rather than the materials used for the ECMO circuit.

Even when venous drainage is ideal and when little or no mixing of blood occurs, a standard ECMO circuit may not be able to provide enough flow to achieve adequate oxygenation in patients with very high cardiac output. Despite its potential limitations, the use of a second circuit in parallel with the first should be considered when other methods of managing refractory hypoxemia during VV ECMO are impossible to implement or unsuccessful.

\section{References}

1. Montisci A, Maj G, Zangrillo A, Winterton D, Pappalardo F. Management of refractory hypoxemia during venovenous extracorporeal membrane oxygenation for ARDS. ASAIO J. 2015;61:227-36.

2. Guarracino F, Zangrillo A, Ruggeri L, Pieri M, Calabrò MG, Landoni G, et al $\beta$-Blockers to optimize peripheral oxygenation during extracorporeal membrane oxygenation: a case series. J Cardiothorac Vasc Anesth. 2012;26:58-63.

3. Bonacchi M, Harmelin G, Peris A, Sani G. A novel strategy to improve systemic oxygenation in venovenous extracorporeal membrane oxygenation: the " $\chi$-configuration". J Thorac Cardiovasc Surg. 2011;142:1197-204.

4. Squiers JJ, Lima B, DiMaio JM. Contemporary extracorporeal membrane oxygenation therapy in adults: Fundamental principles and systematic review of the evidence. J Thorac Cardiovasc Surg. 2016;152:20-32.

5. Huang CT, Tsai YJ, Tsai PR, Ko WJ. Extracorporeal membrane oxygenation resuscitation in adult patients with refractory septic shock. J Thorac Cardiovasc Surg. 2013;146:1041-6.

6. ELSO guidelines for cardiopulmonary extracorporeal life support. Version 1.3. Available at: www.elsonet.org. Accessed September 16, 2016. 